\title{
Trends in qualifying biomarkers in drug safety. Consensus of the 2011 meeting of the Spanish Society of Clinical Pharmacology
}

\author{
José A. G. Agúndez ${ }^{1,2}$ *, Jaime del Barrio ${ }^{3}$, Teresa Padró ${ }^{4,5}$, Camilla Stephens ${ }^{6,7}$, Magí Farré $^{8}$, \\ Raúl J. Andrade ${ }^{7,9}$, Lina Badimon ${ }^{4,5}$, Elena García-Martín ${ }^{2,10}$, Gemma Vilahur $^{4,5}$ and M. Isabel Lucena ${ }^{6,7}$ \\ 1 Department of Pharmacology, University of Extremadura, Cáceres, Spain \\ ${ }^{2}$ Red de Investigación de Reacciones Adversas a Alergenos y Fármacos, Cáceres, Spain \\ ${ }^{3}$ Roche Institute, Madrid, Spain \\ ${ }^{4}$ Cardiovascular Research Center, CSIC-ICCC, Biomedical Research Institute Sant-Pau, Barcelona, Spain \\ ${ }^{5}$ Centro de Investigaciones Biomédicas en Red de Fisiopatología de la Obesidad y Nutrición, Madrid, Spain \\ ${ }^{6}$ Clinical Pharmacology Service, Facultad de Medicina, Hospital Universitario Virgen de la Victoria, Málaga, Spain \\ ${ }^{7}$ Centro de Investigaciones Biomédica en Red de Enfermedades Hepáticas y Digestivas, Barcelona, Spain \\ ${ }^{8}$ Human Pharmacology, Hospital del Mar Research Institute-IMIM, Universidad Autónoma de Barcelona, Barcelona, Spain \\ ${ }^{9}$ Liver Unit, Facultad de Medicina, Hospital Universitario Virgen de la Victoria, Málaga, Spain \\ ${ }^{10}$ Department of Biochemistry and Molecular Biology, University of Extremadura, Cáceres, Spain
}

\section{Edited by:}

Andrea Gaedigk, The Children's Mercy

Hospital and Clinics, USA

Reviewed by:

Todd Skaar, Indiana University Medical Center, USA

Larisa Humma Cavallari, University of Illinois at Chicago, USA

*Correspondence:

José A. G. Agúndez, Department of Pharmacology, University of

Extremadura, Avda de la Universidad s/n, E-10071 Cáceres, Spain.

e-mail: jagundez@unex.es
In this paper we discuss the consensus view on the use of qualifying biomarkers in drug safety, raised within the frame of the XXIV meeting of the Spanish Society of Clinical Pharmacology held in Málaga (Spain) in October, 2011. The widespread use of biomarkers as surrogate endpoints is a goal that scientists have long been pursuing. Thirty years ago, when molecular pharmacogenomics evolved, we anticipated that these genetic biomarkers would soon obviate the routine use of drug therapies in a way that patients should adapt to the therapy rather than the opposite. This expected revolution in routine clinical practice never took place as quickly nor with the intensity as initially expected. The concerted action of operating multicenter networks holds great promise for future studies to identify biomarkers related to drug toxicity and to provide better insight into the underlying pathogenesis. Today some pharmacogenomic advances are already widely accepted, but pharmacogenomics still needs further development to elaborate more precise algorithms and many barriers to implementing individualized medicine exist. We briefly discuss our view about these barriers and we provide suggestions and areas of focus to advance in the field.

Keywords: biomarkers, pharmacogenomics, drug-induced liver injury, drug safety

\section{INTRODUCTION}

The widespread use of genetic and non-genetic biomarkers as surrogate endpoints is a goal that scientists have long been pursuing, aiming to describe risks, exposures, intermediate effects of treatments, and biologic mechanisms. Biomarkers are measurements that indicate biological processes, and can be used in clinical practice for disease diagnosis, prognosis, therapy selection, dose adjustment, and monitoring outcomes. Key issues are the identification of high-risk cohorts and biomarkers that can predict a response to a particular intervention. Whereas a clinical endpoint is a characteristic or variable that reflects how a patient feels, functions, or survives Biomarkers Definitions Working Group (2001), a surrogate endpoint is a biomarker that is intended to substitute for a clinical endpoint. That is, a characteristic that is objectively measured and evaluated as an indicator of normal biologic processes, pathogenic processes, or pharmacologic responses to a therapeutic intervention (Biomarkers Definitions Working Group, 2001). These biomarkers are expected to predict the clinical response based on epidemiological, therapeutical, pathophysiological, or other scientific evidence. In relation to its linkage to efficacy or safety outcomes, three forms of biomarkers can be defined: a target biomarker measures physical or biological interactions with the molecular target; these target biomarkers are usually part of the "proof of mechanism" in drug development. A second type of biomarkers, defined as mechanism biomarkers, measures a biological effect presumed to be downstream of the target. Finally, outcome biomarkers substitute for a clinical outcome or predict an outcome of a disease or toxicity following treatment. Biomarkers can be of five types: physiological measurements, blood tests or other chemical analyses of tissue or bodily fluids, genetic data, metabolic data, and measurements obtained from images (Institute of Medicine, 2011).

In recent years the developments in genetic biomarkers have moved from studying the genome sequence and structure to assessing interindividual variability and trying to understand how genetic biomarkers relate with the pathophysiology of human diseases and the outcome of drug therapies, particularly in drug efficacy and toxicity. The development of these genetic biomarkers 
to stratify patient populations (patient selection biomarkers) into those who should or should not receive a given drug has been extensively investigated in recent years, and is one of the main instruments of "personalized medicine" (PHG Foundation, 2010; Green and Guyer, 2011). Nevertheless until now only a small number of biomarkers are being used routinely in clinical practice. Several barriers to implementing the use of these biomarkers exist (see Table 1 for some examples). Some of these barriers will be discussed below. We should note that biomarker qualification has not been covered in any International Conference of Harmonization (ICH) guideline except for the genomic biomarkers (European Medicines Agency, 2009). Initiatives such as the safer and faster evidence-based translation (SAFE-T) or the cooperative research thematic network on adverse reactions to allergens and drugs in Spain (RIRAAF), which focus on both genetic and non-genetic biomarkers, were designed to provide robust markers on drug safety.

The use of biomarkers requires a wide consensus and the development of adequate guidelines of its use and implementation. This manuscript summarizes the view of the participants regarding barriers to implementing the use of biomarkers in clinical practice, as well as suggestions and areas of focus. This consensus view was elaborated within the frame of the roundtable of Biomarkers in the XXIV meeting of the Spanish Society of Clinical Pharmacology held in Málaga (Spain) in October, 2011. Details

\section{Table 1 | Examples of barriers to implementing individualized medicine.}

\section{BARRIERS DISCUSSED INTHIS PAPER}

Need for demonstration of clinical validity and utility

Need for clear guidelines for the use of biomarkers in clinical medicine

Insufficient development of processes and protocols required to translate

biomarker information to clinical practice

- Need for more precise algorithms

- Implementing pharmacogenomics with other "omics"

- Use of surrogate tag-SNPs

- The need of implementation of gene/drug pairs

The need for specific biomarkers for severe ADRs

Concerns about test costs

The need for the identification of new biomarkers

\section{BARRIERS DISCUSSED ELSEWHERE}

Fragmentation of health-care systems

Low use of electronic medical records

Health-care systems do not reward the prevention of ADRs

Insufficient awareness about genomics among clinicians

Little genetic testing is done preemptively

Lack of a clear assessment of ethical, legal, and social implication of biomarker testing

These examples may not apply to all countries. For instance, in some countries electronic records may be under-utilized or not existing. In other countries the use of electronic records is compulsory in public health assistance.

Compiled from this study and other studies published elsewhere (Deverka et al., 2007; Haga and Burke, 2008; Agundez et al., 2009; Relling et al., 2010; Matheis et al., 2011; Relling and Klein, 2011). about the meeting, program, and participants can be accessed in the webpage ${ }^{1}$.

\section{USE OF BIOMARKERS IN THE ASSESSMENT OF ADVERSE DRUG REACTIONS}

Serious adverse effects are a major challenge in current drug treatment and a major cause for drug withdrawal from the pharmaceutical market. Insufficient development of specific biomarkers makes causality assessment challenging, is a barrier for the prediction of susceptible patients and hurdles the development of safer new drugs. Thirty years ago, when molecular pharmacogenomics evolved, we shared the hope that these genetic biomarkers would soon allow the routine use of drug therapies adapted to every patient and to every condition. This expected transformation in routine clinical practice never came about neither as quickly nor with the intensity as initially expected.

The conversion of genomic data into clinically useful information on how individuals vary in their response to drugs (that is, the demonstration of clinical validity and utility) still needs further development (Lesko et al., 2010). Thiopurine methyltransferase (TPMT) is a good example of this. Whereas a strong phenotype/genotype association exists for TPTM (Ford et al., 2009) most of the azathioprine/mercaptopurine-induced adverse reactions and the efficacy of therapy are not explained by TPMT gene polymorphisms (Palmieri et al., 2007). It seems that pharmacogenomics alone is not sufficient to explain all the interindividual variability in drug response, and in recent years many articles have been published indicating lack of association between polymorphisms in genes coding for drug-metabolizing enzymes and drugs response, even though these drugs are substrates for the enzymes encoded by the target genes. From an initial point of view of pharmacogenomics and adverse drug reactions (ADRs), alterations in genes coding for drug-metabolizing enzymes would cause a change in the metabolic capacity resulting in accumulation (or lower levels) of the drug. These alterations in drug concentration would then change the drug response. This initial view has turned out to be too naive for most drugs used in clinical practice. Many drugs initially thought to be specific substrates for an enzyme, actually follow several metabolic pathways (Pachkoria et al., 2007). Rapidly increasing evidence indicates that the term enzyme-specific to define a drug should be used cautiously (Agundez et al., 2009). Moreover, many mutations in genes coding for drug-metabolizing enzymes are not actually linked to major changes in enzyme activity.

Recently in the World PGX Summit held in Boston, the question of whether we have made significant progress or got stuck with a few examples was proposed (Groenen, 2011). The conclusion suggests that we have been somewhat overoptimistic. Many predictions have come true, but many years later than predicted (Groenen, 2011). Today some pharmacogenomic advances are already widely accepted but the available information is still insufficient: The Food and Drug Administration (FDA) has published a "Table of Pharmacogenomic Biomarkers in Drug Label," that recognizes the role of pharmacogenomics in identifying responders

${ }^{1}$ http://www.sefc2011.com/index.php? $\mathrm{m}=$ content $\& \mathrm{e}=44 \& \mathrm{catid}=2 \&$ PHPSESSID $=6347 \mathrm{f} 60 \mathrm{e} 702128 \mathrm{bceadc} 3 \mathrm{~b} 6 \mathrm{~d} 7853 \mathrm{ae} 86$ 
and non-responders to medications, avoiding adverse events, and optimizing drug dose (Food and Drug Administration, 2011). The Clinical Pharmacogenetics Implementation Consortium (CPIC) of the National Institutes of Health's Pharmacogenomics Research Network $^{2}$ and the Pharmacogenomics Knowledge Base (Phar$\mathrm{mGKB})^{3}$, as well as other national and international initiatives, are particularly active in providing guidelines and therapeutic recommendations based on pharmacogenomic testing (Relling and Klein, 2011; Swen et al., 2011). Table 2 summarizes some major gene targets proposed in these initiatives. Although it should be emphasized that discrepancies among therapeutic recommendations exist. The development of pharmacogenomic guidelines and recommendations within the frame of a Spanish consortium is an ongoing project.

Today we have pharmacogenomic-based recommendations in clinical practice for a very limited number of drugs (about 1-2\% of drugs on the market). In addition, the discrimination capacity of pharmacogenomics tests within different categories of patients is limited. That is, all individuals with the same number of defect alleles are classified in a single group, whereas evidences for a large interindividual variability in the metabolic capacity among individuals with the same genotype exist. At best, pharmacogenomicbased recommendations usually fall within one of the following categories: "no action required; select an alternative drug; reduce the dose by a determined percentage; or monitor plasma concentration." For instance, for phenytoin therapy it is recommended to reduce the maintenance dose by 25 and $50 \%$ for individuals heterozygotes and homozygotes, respectively, for CYP2C9 defect alleles (Swen et al., 2011). Although this is an improvement on the pre-pharmacogenomics situation, we should ask ourselves if classifying patients in three categories, instead of one, is enough improvement. An additional effort to elaborate more precise algorithms predicting drug response is urgently required. For the future development of such algorithms it would be necessary to include many factors besides polymorphisms of genes coding for drug-metabolizing enzymes. These should include at least factors related to gene expression and regulation, such as the patient's transcriptomics signature, phenome, and metabolic profile with a given drug including relevant lifestyle and environmental factors that influence drug metabolism or drug response.

In the last years it has become relatively common to use tag-SNPs, many of which do not actually cause any functional alteration in drug-metabolizing enzymes, as a surrogate marker for another surrogate marker that is, the single nucleotide polymorphism (SNP) which actually causes the alteration in drug metabolism. The use of these surrogates of surrogates can be of some use from a practical point of view, for instance, to reduce the cost of the genetic analyses (Garcia-Closas et al., 2011). However, the price that we have to pay for this relatively small reduction in costs (or in time) is a reduction in the predictive capacity of the biomarkers (Agundez et al., 2008; Selinski et al., 2011). As mentioned earlier, increasing the predictive capacity of biomarkers is one of the major problems that we have to solve to translate the

${ }^{2}$ http://www.pgrn.org

${ }^{3}$ http://www.pharmgkb.org

Table 2 | Examples of pharmacogenomic targets with therapeutic recommendations.

Gene/marker Drug involved

\section{IL28B}

$5 \mathrm{q}$ Chromosome

ALK

BRAF

CCR5

CD30

Cholinesterase gene

CYP1A2

CYP2C19

CYP2C9

CYP2D6

$D P D$

EGFR

ER receptor

G6PD

Her2/neu

HLA-B*1502

HLA- $B * 5701$

LDL receptor

NAT1/NAT2

Ph Chromosome

PML/RAR $\alpha$

Rh genotype

TPMT

UGT1A1

VKORC1
Boceprevir, peginterferon alfa-2b, telaprevir

Lenalidomide

Crizotinib

Vemurafenib

Maraviroc

Brentuximab, vedotin

Mivacurium

Dexlansoprazole

Citalopram, clopidogrel, dexlansoprazole, diazepam, esomeprazole, imipramine, lansoprazole, nelfinavir, omeprazole, pantoprazole, prasugrel, rabeprazole, sertraline, ticagrelor, voriconazole

Acenocoumarol, carisoprodol, carvedilol, celecoxib, flurbiprofen, phenytoin, warfarin

Amitriptyline, aripiprazole, atomoxetine, cevimeline, citalopram, clomipramine, clozapine, codeine, desipramine, desloratadine, dextromethorphan, doxepin, flecainide, fluoxetine, galantamine, gefitinib, haloperidol, iloperidone, imipramine, metoprolol, nortriptyline, oxycodone, paroxetine, perphenazine, pimozide, propafenone, propranolol, protriptyline, quinidine, risperidone, tetrabenazine, thioridazine, timolol, tiotropium, tolterodine, tramadol, trimipramine, venlafaxine, zuclopenthixol

Fluorouracil, capecitabine, tegafur

Cetuximab, erlotinib, gefitinib, panitumumab

Fulvestrant, tamoxifen

Chloroquine, dapsone, rasburicase

Lapatinib, trastuzumab

Carbamazepine

Abacavir

Atorvastatin

Hydralazine, isoniazid, isosorbide, pyrazinamide, rifampin

Busulfan, dasatinib, nilotinib

Arsenic trioxide, tretinoin

Clomiphene

Azathioprine, mercaptopurine, thioguanine

Irinotecan

Acenocoumarol, phenprocoumon

Compiled from (Food and Drug Administration, 2011; Johnson et al., 2011; Relling et al., 2011; Scott et al., 2011; Swen et al., 2011) and CPIC Gene-Drug Pairs (see http://www.pharmgkb.org/contributors/consortia/cpic_gene-drug_pairs.jsp).

advances in pharmacogenomics to routine clinical practice. The new platforms have dramatically reduced the cost of genotyping and the use of surrogates of surrogates is presently no longer justified even in terms of cost-benefit.

The development of specific biomarkers for severe ADRs such as idiosyncratic drug-induced liver toxicity is particularly urgent. As the liver plays a central role in drug metabolism, nearly every 
prescribed medication comes with a potential threat of hepatotoxicity. Despite being a relatively rare condition drug-induced liver injury (DILI) can lead to serious health problems, with bearings on public health and during preclinical and clinical drug development. In fact, DILI remains a significant cause of market withdrawal of commercialized drugs or adoption of regulatory measures despite rigorous preclinical and clinical evaluation processes. Subsequently, the liver is today the major target organ for toxicity assessments in the course of drug research and development. Despite increasing attention to the impact and pathology of DILI during the last decade, the underlying mechanism of DILI development is still mainly undefined, partly due to the lack of a functional animal model in idiosyncratic hepatotoxicity. However, it is accepted that genetic variations play an important role in determining hepatotoxicity susceptibility. Early pharmacogenetic studies on DILI were mainly conducted using the candidate gene approach (CGA), whereby potential genes were selected based on the understanding of their cellular role and then analyzed for putative genetic variation between cases and controls. Many of these studies, therefore, focused on genes involved in drug metabolism and excretion. This resulted in findings of associations between DILI susceptibility and several polymorphisms in various genes, such as specific alleles in manganese superoxide dismutase, glutathione peroxidase, and glutathione $S$-transferase that are significantly more frequent in DILI patients than in controls and shared across multiple drugs (Lucena et al., 2008, 2010; Andrade et al., 2009; Agundez et al., 2011). Genome-wide association studies (GWAS), and international projects such as encyclopedia of DNA elements (ENCODE) or the 1000 Genomes project, all still under way, are providing us with a more thorough picture of human genetic variability and its relation to drug response. In particular the introduction of the GWAS methodology has opened up a new path for DILI studies. Several DILI GWA studies using SNP arrays have been performed to date focusing on different causal agents. Interestingly, all of these studies have identified SNPs with genome-wide significance located in the major histocompatibility (MHC) region on chromosome 6. In addition, some of the identified SNPs appear to be shared between different forms of DILI and in some cases even between different forms of ADRs, despite the causal agents being functionally and structurally very different (Daly et al., 2009; Lucena et al., 2011b). The fact that most idiosyncratic DILI cases do not demonstrate clinical features of hypersensitivity (present only in a $23 \%$ of the cases; Singer et al., 2010; Lucena et al., 2011b) highlights the potential of GWAS to detect novel targets that may not have been chosen for CGA studies relying on mechanistic strategies. Nevertheless, it should be emphasized that the combined use of GWA and CGA studies is required to achieve a full picture of the genetic factors related to drug safety. GWAS findings can at times be difficult to explain in terms of a plausible biological hypothesis and often are not replicated in further CGAS focusing on the target SNPs detected in the GWAS. Moreover, GWAS are not adequate to characterize copy number variations in candidate genes. The current generation of DNA sequencers produce 12 orders of magnitude more data per run than the most powerful platform available in 2001, and at a much lower price (a few thousand vs. several million Euros). It is therefore expected that the use of the next generation sequencing technologies could increase the potential of CGAS and provide more robust associations between variations in candidate genes identified in GWAS and the risk of developing DILI and other severe ADRs.

Besides technical limitations, many factors hamper a faster development of genetic testing in ADRs. Among these, the lack of sufficient evidence for cost-efficiency is one of the most relevant. Based on average prices, it appears that the cost of implementing a robust, validated diagnostic test into clinical practice should not be, in principle, exorbitant. However, we will need to conduct proper economic evaluation assessments to find out how it compares, in terms of cost-efficiency, with already existing tests, and what savings its implementation might bring about. Biomarkerbased innovations, if assessed and implemented properly, should be a bonus, not an additional burden on health-care budgets.

The need for the identification of new biomarkers for drug toxicity and response is another key point that should be addressed in the next years. The SAFE-T initiative proposes a generic qualification strategy for new translational safety biomarkers to enable potential new medicines to be evaluated more quickly and safely in patients. The project, run by a public-private partnership of pharmaceutical industry, academics, and small-medium enterprises, addresses the current lack of sensitive and specific clinical tests to diagnose and monitor drug-induced injury of the kidney, liver, and vascular system, which are a major hurdle in drug development (Matheis et al., 2011). SAFE-T operates under the framework of the EU Innovative Medicines Initiative Joint Undertaking (IMI-JU) ${ }^{4}$. First, potential biomarker candidates for drug-induced injury of the kidney, liver, and vascular system were evaluated and prioritized for clinical qualification. The biomarkers (proteins, metabolites, RNA), once approved by regulatory agencies for prediction, diagnosis, and monitoring of organ injuries in a drug development context, could also be used for diagnosing renal, hepatic, and vascular diseases in a clinical setting.

\section{FUTURE DEVELOPMENTS}

Three major factors are providing momentum for the development of personalized medicine. First, the large variability of current medicines among patients (both in terms of efficacy and safety), which entails considerable human and economic costs. Second, economic pressures ensuing from inefficiencies in drug discovery and development processes, a tripling in pharmaceutical companies' R\&D investments in the last 10 years has not translated into a significant increase in the number of new drug approvals. Finally, technology, in particular genotyping and sequencing technologies, have undergone a spectacular transformation in the last decade becoming much faster, accurate, and, above all accessible not only to large scientific consortia, but also to smaller institutions and research groups.

The concerted action of operating multicenter networks holds great promise for future studies to identify genetic biomarkers related to drug toxicity and to provide better insight into the underlying pathogenesis. Several large networks on ADRs have

${ }^{4}$ http://www.imi.europa.eu 
been set up around the world, such as the Spanish DILI Registry, the RIRAAF network, the drug-induced liver injury network (DILIN) in the US to enhance the identification and diagnosis of DILI cases. Likewise, the International DILI Consortium (IDILIC) has been initiated to enable the performance of high powered genetic studies. African, Latin-American, and some other populations are under-represented in the studies conducted so far, but this is expected to change. With this in mind, the Spanish DILI Registry has recently extended its network into Latin America and created a new branch, the Spanish-Latin DILI Network (Lucena et al., 2011a). Some international initiatives have set up consortia to develop sufficient evidence to qualify new predictive safety tests for regulatory use that may improve the safety of new drugs entering the market (e.g., the Biomarker Consortium ${ }^{5}$, Predictive Safety Testing Consortium ${ }^{6}$, the $\mathrm{CPIC}^{7}$ and the International Serious Adverse Event Consortium - iSAEC) ${ }^{8}$. As part of the Innovative Medicines Initiative in the European Union, the SAFE-T consortium ${ }^{9}$ has compiled a comprehensive clinical program to help identifying and qualifying new safety biomarkers for drug-induced kidney, liver, and vascular injury. It is anticipated that the biomarker and genomic era will allow us to refine definitions of risk, predict response to a particular intervention, guide

\footnotetext{
${ }^{5}$ www.biomarkersconsortium.org

${ }^{6}$ www.c-path.org/pstc.cfm

${ }^{7}$ http://www.pharmgkb.org/contributors/consortia/cpic_profile.jsp

${ }^{8}$ www.saeconsortium.org

${ }^{9}$ www.imi-safe-t.eu
}

\section{REFERENCES}

Agundez, J. A., Garcia-Martin, E., and Martinez, C. (2009). Genetically based impairment in CYP2C8- and CYP2C9-dependent NSAID metabolism as a risk factor for gastrointestinal bleeding: is a combination of pharmacogenomics and metabolomics required to improve personalized medicine? Expert Opin. Drug Metab. Toxicol. 5, 607-620.

Agundez, J. A., Golka, K., Martinez, C., Selinski, S., Blaszkewicz, M., and Garcia-Martin, E. (2008). Unraveling ambiguous NAT2 genotyping data. Clin. Chem. 54, 1390-1394.

Agundez, J. A., Lucena, M. I., Martinez, C., Andrade, R. J., Blanca, M., Ayuso, P., and Garcia-Martin, E. (2011). Assessment of nonsteroidal antiinflammatory drug-induced hepatotoxicity. Expert Opin. Drug Metab. Toxicol. 7, 817-828.

Andrade, R. J., Agundez, J. A., Lucena, M. I., Martinez, C., Cueto, R., and Garcia-Martin, E. (2009). Pharmacogenomics in drug induced liver injury. Curr. Drug Metab. 10, 956-970.

Biomarkers Definitions Working Group. (2001). Biomarkers and surrogate endpoints: preferred definitions and conceptual framework. Clin. Pharmacol. Ther. 69, 89-95.
Daly, A. K., Donaldson, P. T., Bhatnagar, P., Shen, Y., Pe'er, I., Floratos, A., Daly, M. J., Goldstein, D. B., John, S., Nelson, M. R., Graham, J., Park, B. K., Dillon, J. F., Bernal, W., Cordell, H. J., Pirmohamed, M., Aithal, G. P., and Day, C. P. (2009). HLA$\mathrm{B} * 5701$ genotype is a major determinant of drug-induced liver injury due to flucloxacillin. Nat. Genet. 41, 816-819.

Deverka, P. A., Doksum, T., and Carlson, R. J. (2007). Integrating molecular medicine into the US health-care system: opportunities, barriers, and policy challenges. Clin. Pharmacol. Ther. 82, 427-434.

European Medicines Agency. (2009). ICH guideline E16. Genomic Biomarkers Related to Drug Response: Context, Structure and Format of Qualification Submissions, EMA/CHMP/ICH/380636/2009.

London: European Medicines Agency.

Food and Drug Administration. (2011). Table of Pharmacogenomic Biomarkers in Drug Labels. Available at: http://www.fda.gov/ drugs/scienceresearch/researchareas/ pharmacogenetics/ucm083378.htm

Ford, L., Kampanis, P., and Berg, J. (2009). Thiopurine S-methyltransferase

dose selection, and enhance the benefit-risk profile of approved medicines.

Future expectations in this area include the development of new diagnostic tools to improve drug toxicity analysis and to identify susceptible individuals prior to treatment initiation. This translational research effort involving genetic and non-genetic markers will hopefully be incorporated to clinical practice in the near future. Nevertheless, besides the barriers summarized in Table 1, it should be kept in mind that health-care providers and large university hospitals are necessarily going to play a crucial role in the widespread implementation of personalized medicine. To do so, they will have to reassess their organizational models and address the urgent educational needs of health practitioners (clinicians, pharmacists, etc., but also managers and decision-makers at the higher levels) in genomic medicine.

\section{ACKNOWLEDGMENTS}

The work in the author's laboratory is financed by Grants PS09/00943, PS09/00469, PS09/01384, FIS-FEDERPI081913, RETICS RIRAAF RD07/0064/0016, REDINSCOR RD06/0003/0015, TERCEL RD06/010/0017, RTA RD06/0001/1009, CIBERehD and CIBERobn from Instituto de Salud Carlos III, Madrid, Spain, and by Grants SAF10/16549 from Ministerio de Ciencia y Tecnología, CTS-6470 from Consejeria de Salud, Junta de Andalucía, Spain, and GR10068 from Junta de Extremadura, Spain. Financed in part with FEDER funds from the European Union. IMI-JU is partly funding the research activities under the SAFE-T project (Grant Agreement No 115003).

genotype-phenotype concordance: used as a quality assurance tool to help control the phenotype assay. Ann. Clin. Biochem. 46, 152-154.

Garcia-Closas, M., Hein, D. W., Silverman, D., Malats, N., Yeager, M., Jacobs, K., Doll, M. A., Figueroa, J. D., Baris, D., Schwenn, M., Kogevinas, M., Johnson, A., Chatterjee, N., Moore, L. E., Moeller, T., Real, F. X., Chanock, S., and Rothman, N. (2011). A single nucleotide polymorphism tags variation in the arylamine $\mathrm{N}$-acetyltransferase 2 phenotype in populations of European background. Pharmacogenet. Genomics 21, 231-236.

Green, E. D., and Guyer, M. S. (2011). Charting a course for genomic medicine from base pairs to bedside. Nature 470, 204-213.

Groenen, P. M. (2011). Conference scene: four decades of modern pharmacogenomics: from promise to clinical utility. Pharmacogenomics 12, 1249-1252.

Haga, S. B., and Burke, W. (2008). Pharmacogenetic testing: not as simple as it seems. Genet. Med. 10, 391-395.

Institute of Medicine. (2011). Perspectives on Biomarker and Surrogate Endpoint Evaluation: Discussion Forum Summary. Washington, DC: The National Academies Press.
Johnson, J. A., Gong, L., Whirl-Carrillo, M., Gage, B. F., Scott, S. A., Stein, C. M., Anderson, J. L., Kimmel, S. E., Lee, M. T., Pirmohamed, M., Wadelius, M., Klein, T. E., and Altman, R. B. (2011). Clinical pharmacogenetics implementation consortium guidelines for CYP2C9 and VKORC1 genotypes and warfarin dosing. Clin. Pharmacol. Ther. 90, 625-629.

Lesko, L. J., Zineh, I., and Huang, S. M. (2010). What is clinical utility and why should we care? Clin. Pharmacol. Ther. 88, 729-733.

Lucena, M. I., Andrade, R. J., Martinez, C., Ulzurrun, E., Garcia-Martin, E., Borraz, Y., Fernandez, M. C., Romero-Gomez, M., Castiella, A., Planas, R., Costa, J., Anzola, S., and Agundez, J. A. (2008). Glutathione S-transferase $\mathrm{ml}$ and $\mathrm{t} 1$ null genotypes increase susceptibility to idiosyncratic drug-induced liver injury. Hepatology 48, 588-596.

Lucena, M. I., Cohen, H., Hernandez, N., Bessone, F., Dacoll, C., Stephens, C., Borraz, Y., Ulzurrun, E., Bruguera, M., and Andrade, R. J. (2011a). Hepatotoxicity, a global problem with local features: toward the creation of a Pan-American hepatotoxicity network. Gastroenterol. Hepatol. 34, 361-368. 
Lucena, M. I., Molokhia, M., Shen, Y., Urban, T. J., Aithal, G. P., Andrade, R. J., Day, C. P., Ruiz-Cabello, F., Donaldson, P. T., Stephens, C., Pirmohamed, M., Romero-Gomez, M., Navarro, J. M., Fontana, R. J., Miller, M., Groome, M., Bondon-Guitton, E., Conforti, A., Stricker, B. H., Carvajal, A., Ibanez, L., Yue, Q. Y., Eichelbaum, M., Floratos, A., Pe'er, I., Daly, M. J., Goldstein, D. B., Dillon, J. F., Nelson, M. R., Watkins, P. B., and Daly, A. K. (2011b). Susceptibility to amoxicillin-clavulanateinduced liver injury is influenced by multiple HLA class I and II alleles. Gastroenterology 141, 338-347.

Lucena, M. I., Garcia-Martin, E., Andrade, R. J., Martinez, C., Stephens, C., Ruiz, J. D., Ulzurrun, E., Fernandez, M. C., RomeroGomez, M., Castiella, A., Planas, R., Duran, J. A., De Dios, A. M., Guarner, C., Soriano, G., Borraz, Y., and Agundez, J. A. (2010). Mitochondrial superoxide dismutase and glutathione peroxidase in idiosyncratic drug-induced liver injury. Hepatology 52, 303-312.

Matheis, K., Laurie, D., Andriamandroso, C., Arber, N., Badimon, L., Benain, X., Bendjama, K., Clavier, I., Colman, P., Firat, H., Goepfert, J., Hall, S., Joos, T., Kraus, S., Kretschmer, A., Merz, M., Padro, T., Planatscher, H., Rossi, A., Schneiderhan-Marra, N., Schuppe-Koistinen, I., Thomann, P., Vidal, J. M., and Molac, B. (2011). A generic operational strategy to qualify translational safety biomarkers. Drug Discov. Today 16, 600-608.
Pachkoria, K., Lucena, M. I., RuizCabello, F., Crespo, E., Cabello, M. R., and Andrade, R. J. (2007). Genetic polymorphisms of CYP2C9 and CYP2C19 are not related to drug-induced idiosyncratic liver injury (DILI). Br. J. Pharmacol. 150, 808-815.

Palmieri, O., Latiano, A., Bossa, F., Vecchi, M., D’Inca, R., Guagnozzi, D., Tonelli, F., Cucchiara, S., Valvano, M. R., Latiano, T., Andriulli, A., and Annese, V. (2007). Sequential evaluation of thiopurine methyltransferase, inosine triphosphate pyrophosphatase, and HPRT1 genes polymorphisms to explain thiopurines' toxicity and efficacy. Aliment. Pharmacol. Ther. 26, 737-745.

PHG Foundation. (2010). Public Health in an Era of Genome-Based and Personalised Medicine. Available at: http:// www.phgfoundation.org/file/6616

Relling, M. V., Altman, R. B., Goetz, M. P., and Evans, W. E. (2010). Clinical implementation of pharmacogenomics: overcoming genetic exceptionalism. Lancet Oncol. 11, 507-509.

Relling, M. V., Gardner, E. E., Sandborn, W. J., Schmiegelow, K., Pui, C. H., Yee, S. W., Stein, C. M., Carrillo, M., Evans, W. E., and Klein, T. E. (2011). Clinical Pharmacogenetics Implementation Consortium guidelines for thiopurine methyltransferase genotype and thiopurine dosing. Clin. Pharmacol. Ther. 89, 387-391.

Relling, M. V., and Klein, T. E. (2011). CPIC: clinical pharmacogenetics implementation consortium of the pharmacogenomics research network. Clin. Pharmacol. Ther. 89 , 464-467.

Scott, S. A., Sangkuhl, K., Gardner, E. E., Stein, C. M., Hulot, J. S., Johnson, J. A., Roden, D. M., Klein, T. E. and Shuldiner, A. R. (2011). Clinical pharmacogenetics implementation consortium guidelines for cytochrome P450-2C19 (CYP2C19) genotype and clopidogrel therapy. Clin. Pharmacol. Ther. 90, 328-332.

Selinski, S., Blaszkewicz, M., Lehmann, M. L., Ovsiannikov, D., Moormann O., Guballa, C., Kress, A., Tru, M. C., Gerullis, H., Otto, T., Barski, D., Niegisch, G., Albers, P., Frees, S. Brenner, W., Thuroff, J. W., AngeliGreaves, M., Seidel, T., Roth, G., Dietrich, H., Ebbinghaus, R., Prager, H. M., Bolt, H. M., Falkenstein, M. Zimmermann, A., Klein, T., Reckwitz, T., Roemer, H. C., Lohlein, D. Weistenhofer, W., Schops, W., Hassan Rizvi, S. A., Aslam, M., Banfi, G., Romics, I., Steffens, M., Ekici, A. B., Winterpacht, A., Ickstadt, K., Schwender, H., Hengstler, J. G., and Golka, K. (2011). Genotyping NAT2 with only two SNPs (rs1041983 and rs1801280) outperforms the tagging SNP rs1495741 and is equivalent to the conventional 7-SNP NAT2 genotype. Pharmacogenet. Genomics 21, 673-678.

Singer, J. B., Lewitzky, S., Leroy, E., Yang, F., Zhao, X., Klickstein, L., Wright, T. M., Meyer, J., and Paulding, C. A. (2010). A genome-wide study identifies HLA alleles associated with lumiracoxib-related liver injury. Nat. Genet. 42, 711-714.

Swen, J. J., Nijenhuis, M., De Boer, A., Grandia, L., Maitland-Van
Der Zee, A. H., Mulder, H., Rongen, G. A., Van Schaik, R. H., Schalekamp, T., Touw, D. J., Van Der Weide, J., Wilffert, B., Deneer, V. H., and Guchelaar, H. J. (2011). Pharmacogenetics: from bench to byte - an update of guidelines. Clin. Pharmacol. Ther. 89, 662-673.

Conflict of Interest Statement: The authors declare that the research was conducted in the absence of any commercial or financial relationships that could be construed as a potential conflict of interest.

Received: 29 October 2011; accepted: 05 January 2012; published online: 19 January 2012.

Citation: Agúndez JAG, del Barrio $J$, Padró T, Stephens C, Farré $M$, Andrade RJ, Badimon L, García-Martín E, Vilahur G and Lucena MI (2012) Trends in qualifying biomarkers in drug safety. Consensus of the 2011 meeting of the Spanish Society of Clinical Pharmacology. Front. Pharmacol. 3:2. doi: 10.3389/fphar.2012.00002

This article was submitted to Frontiers in Pharmacogenetics and Pharmacogenomics, a specialty of Frontiers in Pharmacology.

Copyright (c) 2012 Agúndez, del Barrio, Padró, Stephens, Farré, Andrade, Badimon, García-Martín, Vilahur and Lucena. This is an open-access article distributed under the terms of the Creative Commons Attribution Non Commercial License, which permits non-commercial use, distribution, and reproduction in other forums, provided the original authors and source are credited. 